\title{
Reverse Size-Dependent Electrooxidation of Gold Nanoparticles Coated with Alkanethiol Self-Assembled Monolayers
}

\author{
Badri P. Mainali, Dhruba K. Pattadar and Francis P. Zamborini*
}

Department of Chemistry, University of Louisville, Louisville, Kentucky 40292, United States

\section{Supporting Information}
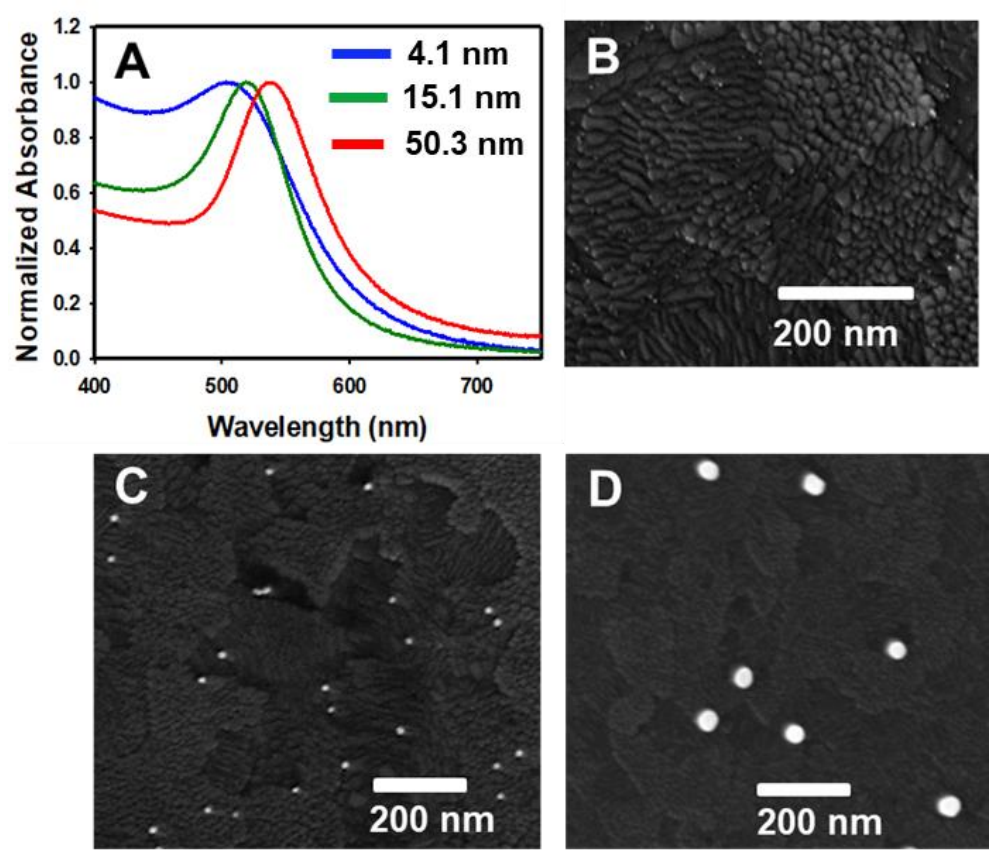

Figure S1. UV-Vis spectra (A) and SEM image of $4.1 \pm 0.7 \mathrm{~nm}(\mathrm{~B})$, $15.1 \pm 1.3 \mathrm{~nm}(\mathrm{C})$ and $50.3 \pm 1.7 \mathrm{~nm}(\mathrm{D})$ diameter citrate-coated $\mathrm{Au}$ NPs attached electrostatically to glass/ITO/APTES. 

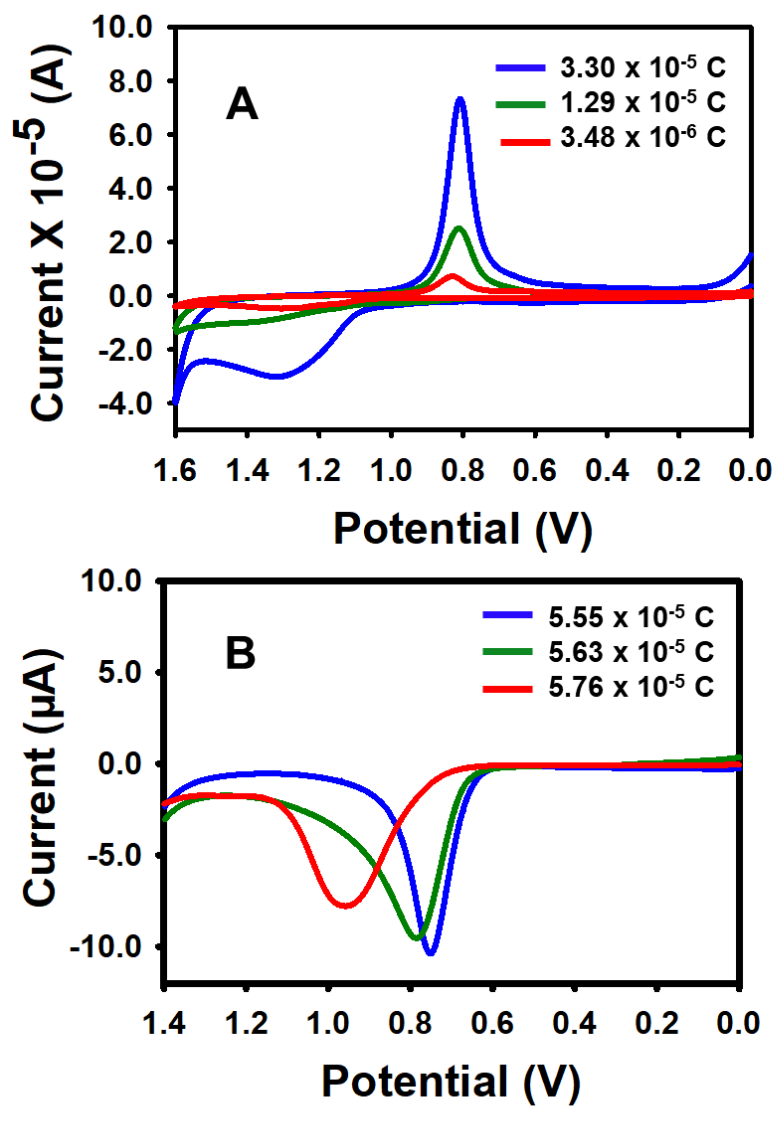

Figure S2. CVs of (A) citrate-coated $4.1 \pm 0.7 \mathrm{~nm}$ (blue), $15.1 \pm 1.3 \mathrm{~nm}$ (green), and $50.3 \pm 1.7$ $\mathrm{nm}$ (red) diameter Au NPs attached to glass/ITO/APTES electrodes obtained in $0.1 \mathrm{M} \mathrm{HClO}_{4}$ at a scan rate of $0.1 \mathrm{~V} / \mathrm{s}$ and (B) ASVs of the same samples in (A) obtained in $0.01 \mathrm{M} \mathrm{KBr}$ plus $0.1 \mathrm{M}$ $\mathrm{KClO}_{4}$ at a scan rate of $0.01 \mathrm{~V} / \mathrm{s}$. This shows the three different sized Au NPs on glass/ITO/APTES with a similar total amount of $\mathrm{Au}(\mathrm{B})$ obtained by soaking in the Au NP solution of interest and monitoring the SA as in (A) until the SA reaches the desired level. 

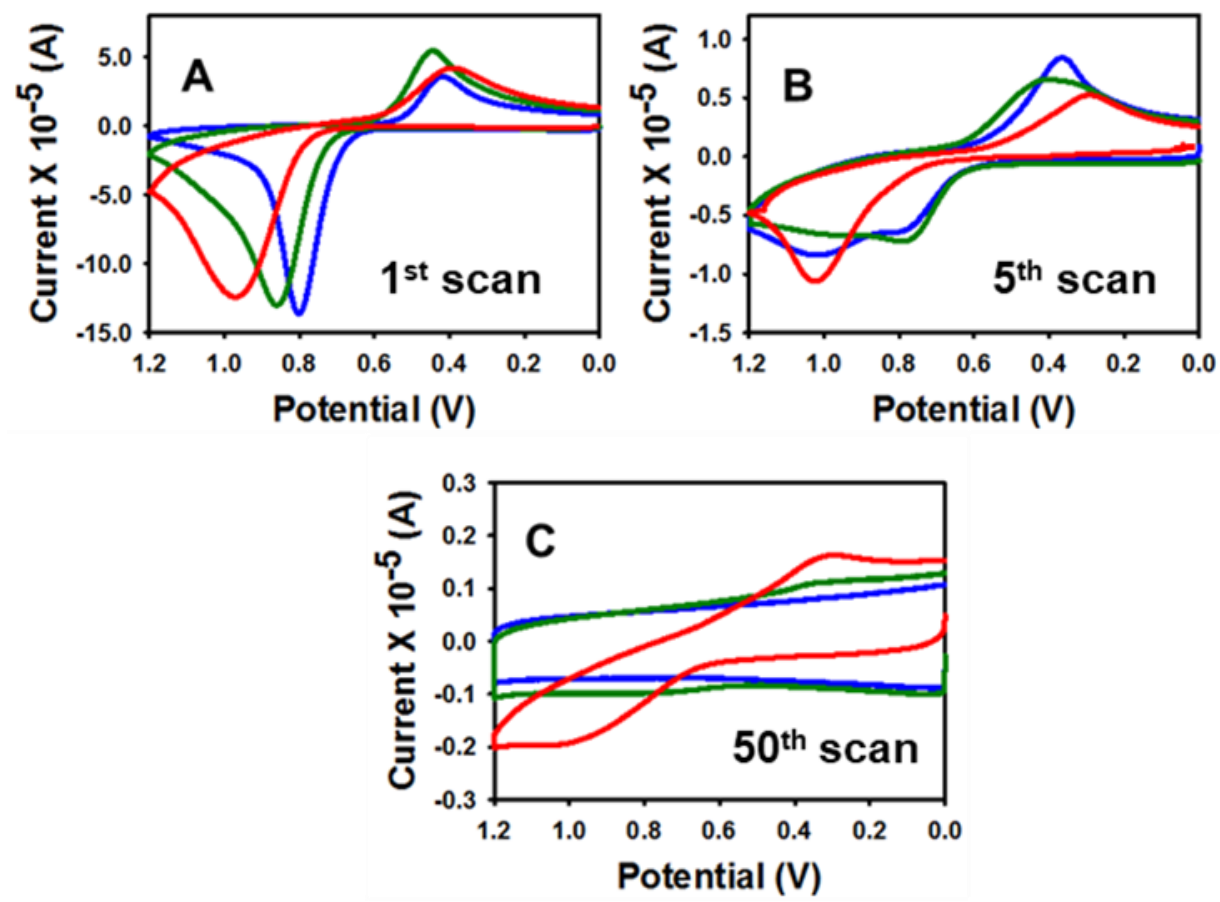

Figure S3. CVs of $4.1 \pm 0.7 \mathrm{~nm}$ (blue), $15.1 \pm 1.3 \mathrm{~nm}$ (green) and $50.3 \pm 1.7 \mathrm{~nm}$ (red) citratecoated Au NPs attached to glass/ITO/APTES obtained in $0.01 \mathrm{M} \mathrm{KBr}$ plus $0.1 \mathrm{M} \mathrm{HClO}_{4}$ at a scan rate of $0.1 \mathrm{~V} / \mathrm{s}$. A $-1^{\text {st }} \mathrm{scan}, \mathrm{B}-5^{\text {th }} \mathrm{scan}, \mathrm{C}-50^{\text {th }}$ scan. 

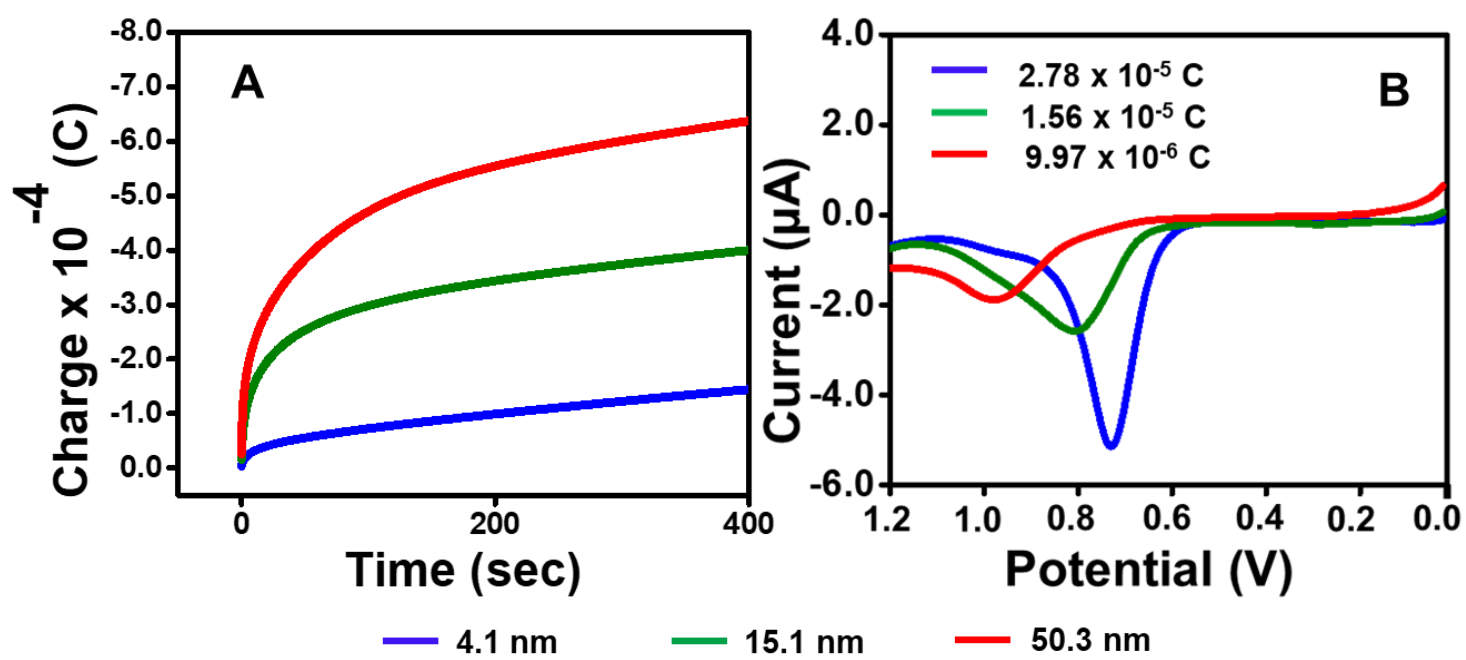

Figure S4. (A) Chronocoulometry (CC) measurements obtained by stepping from $0.0 \mathrm{~V}$ to $1.0 \mathrm{~V}$ and monitoring the charge as a function of time for $400 \mathrm{~s}$ in $0.01 \mathrm{M} \mathrm{KBr}$ plus $0.1 \mathrm{M} \mathrm{HClO}_{4}$ of glass/ITO/APTES electrodes coated with $4.1 \pm 0.7 \mathrm{~nm}$ (blue), $15.1 \pm 1.3 \mathrm{~nm}$ (green), and $50.3 \pm$ $1.7 \mathrm{~nm}$ (red) Au NPs functionalized with C4S SAMs. (B) Anodic stripping voltammetry (ASV) obtained from $0.0 \mathrm{~V}$ to $1.2 \mathrm{~V}$ in $0.01 \mathrm{M} \mathrm{KBr}$ plus $0.1 \mathrm{M} \mathrm{KClO}_{4}$ of the different sized $\mathrm{Au} \mathrm{NPs}$ on glass/ITO/APTES electrodes following the $\mathrm{CC}$ treatment in (A) and $30 \mathrm{~min}$ of ozone treatment. The ASV stripping charge in Coulombs $(\mathrm{C})$ is provided in Frame B corresponding to the different size $\mathrm{Au}$ NPs. The larger peak for Au oxidation of $4.1 \pm 0.7 \mathrm{~nm}$ Au NPs compared to the $15.1 \pm 1.3$ $\mathrm{nm}$ and $50.3 \pm 1.7 \mathrm{~nm} \mathrm{Au} \mathrm{NPs} \mathrm{proves} \mathrm{that} \mathrm{the} \mathrm{C4S} \mathrm{protected} \mathrm{the} \mathrm{smallest} \mathrm{Au} \mathrm{NPs} \mathrm{the} \mathrm{most} \mathrm{from}$ oxidative dissolution by $\mathrm{Br}^{-}$. 

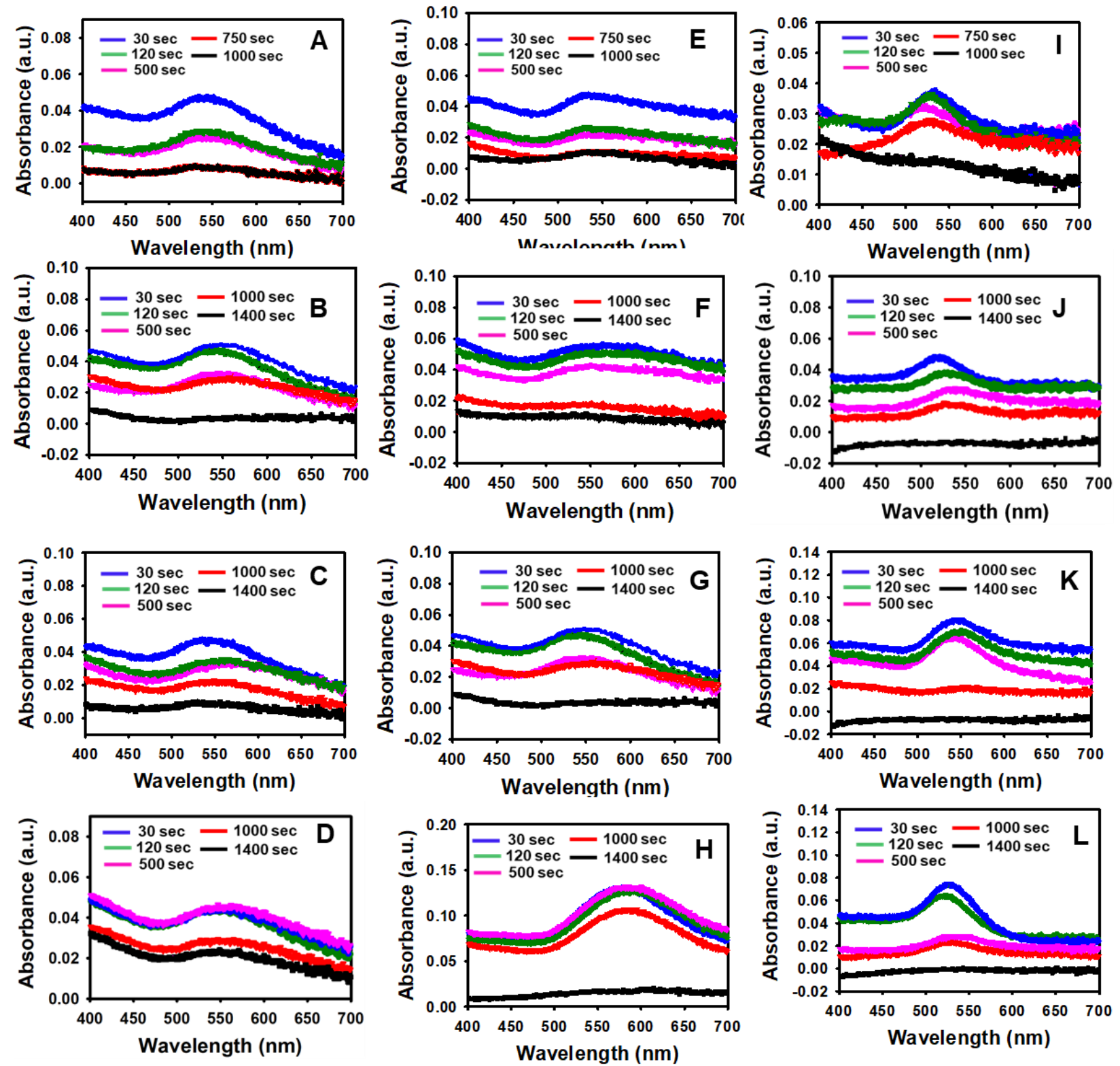

Figure S5. UV-Vis of glass/ITO/APTES attached 4.1 $\pm 0.7 \mathrm{~nm}(\mathrm{~A}-\mathrm{D}), 15.1 \pm 1.3 \mathrm{~nm}(\mathrm{E}-\mathrm{H})$ and $50.3 \pm 1.7 \mathrm{~nm}(\mathrm{I}-\mathrm{L}) \mathrm{Au}$ NPs at different times during $\mathrm{CC}$ in $0.01 \mathrm{M} \mathrm{KBr}$ plus $0.1 \mathrm{M} \mathrm{HClO}_{4}$ at a potential of $1.0 \mathrm{~V}$ up to $1000 \mathrm{sec}$ and at a potential of $1.2 \mathrm{~V}$ from 1000 to $1400 \mathrm{sec}$. Row 1citrate coated $\mathrm{Au}$, row $2-\mathrm{Au} / \mathrm{C} 4 \mathrm{~S}$, row $3-\mathrm{Au} / \mathrm{C} 10 \mathrm{~S}$ and row $4-\mathrm{Au} / \mathrm{C} 16 \mathrm{~S}$. 
Table S1. Integrated charges (Coulombs) of citrate-coated $4.1 \pm 0.7,15.1 \pm 1.3$ and $50.3 \pm 1.7 \mathrm{~nm}$ $\mathrm{Au}$ NPs attached to glass/ITO/APTES electrodes obtained from the Au oxide reduction peak by cyclic voltammetry $(\mathrm{CV})$ in $0.1 \mathrm{M} \mathrm{HClO}_{4}$ at a scan rate of $0.1 \mathrm{~V} / \mathrm{s}$ before performing $\mathrm{CV}$ as in Figure S3A. The oxidation stripping and reduction charge and their averages for the 3 sizes of $\mathrm{Au}$ NPs were also obtained from the $1^{\text {st }} \mathrm{CV}$ scan in $0.01 \mathrm{M} \mathrm{KBr}$ plus $0.1 \mathrm{M} \mathrm{HClO}_{4}$ at a scan rate of $0.1 \mathrm{~V} / \mathrm{s}$.

\begin{tabular}{|c|c|c|c|c|c|c|}
\hline $\begin{array}{c}\text { NPs } \\
\text { diameter } \\
(\mathrm{nm})\end{array}$ & $\begin{array}{l}\text { Number } \\
\text { of trials }\end{array}$ & $\begin{array}{c}\mathrm{CV} \\
\text { coverage } \\
\text { from Au } \\
\text { oxide } \\
\text { reduction } \\
\text { charge in } \\
\mathrm{HClO}_{4}(\mathrm{C})\end{array}$ & $\begin{array}{c}\mathrm{CV} \\
\text { oxidative } \\
\text { stripping } \\
\text { charges } \\
\text { during } 1^{\text {st }} \\
\text { scan in } \mathrm{KBr} \\
\text { (C) }\end{array}$ & $\begin{array}{l}\text { Average of } \\
\text { oxidative } \\
\text { stripping } \\
\text { charges } \\
\text { during } 1^{\text {st }} \\
\text { scan in } \mathrm{KBr} \\
\text { (C) }\end{array}$ & $\begin{array}{c}\mathrm{CV} \\
\text { reduction } \\
\text { charge after } \\
\text { stripping } \\
\text { during } 1^{\text {st }} \\
\text { scan in } \mathrm{KBr} \\
\text { (C) }\end{array}$ & $\begin{array}{l}\text { Average of } \\
\text { reduction } \\
\text { charge after } \\
\text { stripping } \\
\text { during } 1^{\text {st }} \\
\text { scan in } \mathrm{KBr} \\
\text { (C) }\end{array}$ \\
\hline \multirow{3}{*}{$\begin{array}{c}4.1 \\
\pm 0.7\end{array}$} & 1 & $3.33 \times 10^{-5}$ & $7.29 \times 10^{-5}$ & \multirow{3}{*}{$\begin{array}{c}7.64( \pm 0.31) \\
\times 10^{-5}\end{array}$} & $2.32 \times 10^{-5}$ & \multirow{3}{*}{$\begin{array}{c}2.31( \pm 0.10) \\
\times 10^{-5}\end{array}$} \\
\hline & 2 & $3.34 \times 10^{-5}$ & $7.83 \times 10^{-5}$ & & $2.21 \times 10^{-5}$ & \\
\hline & 3 & $3.50 \times 10^{-5}$ & $7.81 \times 10^{-5}$ & & $2.40 \times 10^{-5}$ & \\
\hline \multirow{3}{*}{$\begin{array}{r}15.1 \\
\pm 1.3\end{array}$} & 1 & $1.37 \times 10^{-5}$ & $8.49 \times 10^{-5}$ & \multirow{3}{*}{$\begin{array}{c}7.73( \pm 1.14) \\
\times 10^{-5}\end{array}$} & $3.66 \times 10^{-5}$ & \multirow{3}{*}{$\begin{array}{c}3.54( \pm 0.39) \\
\times 10^{-5}\end{array}$} \\
\hline & 2 & $1.38 \times 10^{-5}$ & $8.27 \times 10^{-5}$ & & $3.86 \times 10^{-5}$ & \\
\hline & 3 & $1.00 \times 10^{-5}$ & $6.48 \times 10^{-5}$ & & $3.11 \times 10^{-5}$ & \\
\hline \multirow{3}{*}{$\begin{array}{l}50.3 \\
\pm 1.7\end{array}$} & 1 & $3.50 \times 10^{-6}$ & $8.15 \times 10^{-5}$ & \multirow{3}{*}{$\begin{array}{c}8.02( \pm 0.78) \\
\times 10^{-5}\end{array}$} & $3.01 \times 10^{-5}$ & \multirow{3}{*}{$\begin{array}{c}3.24( \pm 0.25) \\
\times 10^{-5}\end{array}$} \\
\hline & 2 & $3.27 \times 10^{-6}$ & $8.73 \times 10^{-5}$ & & $3.23 \times 10^{-5}$ & \\
\hline & 3 & $3.92 \times 10^{-6}$ & $7.18 \times 10^{-5}$ & & $3.48 \times 10^{-5}$ & \\
\hline
\end{tabular}


Table S2. Integrated charges (Coulombs) of Au NPs attached to glass/ITO/APTES electrodes obtained from the Au oxide reduction peak by $\mathrm{CV}$ in $0.1 \mathrm{M} \mathrm{HClO}_{4}$ for the different samples of 4.1 $\pm 0.7,15.1 \pm 1.3$ and $50.3 \pm 1.7 \mathrm{~nm}$ Au NPs. The measured charge is proportional to total surface area (SA), which allowed a calculation of total volume $(\mathrm{V})$ based on the known SA/V ratio of 0.64 , 0.23 and 0.060 for $4.1 \pm 0.7,15.1 \pm 1.3$ and $50.3 \pm 1.7 \mathrm{~nm} \mathrm{Au} \mathrm{NPs,} \mathrm{respectively} \mathrm{(no} \mathrm{ozone}$ treatment). Based on this, we maintained a similar amount of total Au atoms on the electrode surface for the different-sized Au NPs for the CV and CC experiments in this work.

\begin{tabular}{|c|c|c|c|c|c|}
\hline \multicolumn{2}{|c|}{$4.1 \pm 0.7 \mathrm{~nm}$} & \multicolumn{2}{|c|}{$15.1 \pm 1.3 \mathrm{~nm}$} & \multicolumn{2}{|c|}{$50.3 \pm 1.7 \mathrm{~nm}$} \\
\hline $\mathrm{CV}(\mathrm{C})$ & $\begin{array}{l}\text { Calculated } \\
\text { ASV (C) }\end{array}$ & $\mathrm{CV}(\mathrm{C})$ & $\begin{array}{l}\text { Calculated } \\
\text { ASV (C) }\end{array}$ & $\mathrm{CV}(\mathrm{C})$ & $\begin{array}{l}\text { Calculated } \\
\text { ASV (C) }\end{array}$ \\
\hline $3.36 \times 10^{-5}$ & $5.25 \times 10^{-5}$ & $1.44 \times 10^{-5}$ & $6.26 \times 10^{-5}$ & $3.50 \times 10^{-6}$ & $5.83 \times 10^{-5}$ \\
\hline $3.29 \times 10^{-5}$ & $5.14 \times 10^{-5}$ & $1.29 \times 10^{-5}$ & $5.61 \times 10^{-5}$ & $3.27 \times 10^{-6}$ & $5.45 \times 10^{-5}$ \\
\hline $3.59 \times 10^{-5}$ & $5.61 \times 10^{-5}$ & $1.38 \times 10^{-5}$ & $6.00 \times 10^{-5}$ & $3.62 \times 10^{-6}$ & $6.03 \times 10^{-5}$ \\
\hline $3.10 \times 10^{-5}$ & $4.84 \times 10^{-5}$ & $1.17 \times 10^{-5}$ & $5.09 \times 10^{-5}$ & $3.55 \times 10^{-6}$ & $5.92 \times 10^{-5}$ \\
\hline $3.04 \times 10^{-5}$ & $4.69 \times 10^{-5}$ & $1.19 \times 10^{-5}$ & $5.17 \times 10^{-5}$ & $2.91 \times 10^{-6}$ & $4.85 \times 10^{-5}$ \\
\hline $3.42 \times 10^{-5}$ & $5.35 \times 10^{-5}$ & $1.06 \times 10^{-5}$ & $4.61 \times 10^{-5}$ & $3.45 \times 10^{-6}$ & $5.75 \times 10^{-5}$ \\
\hline $3.45 \times 10^{-5}$ & $5.39 \times 10^{-5}$ & $0.99 \times 10^{-5}$ & $4.30 \times 10^{-5}$ & $3.16 \times 10^{-6}$ & $5.27 \times 10^{-5}$ \\
\hline $3.40 \times 10^{-5}$ & $5.31 \times 10^{-5}$ & $1.24 \times 10^{-5}$ & $5.39 \times 10^{-5}$ & $3.58 \times 10^{-6}$ & $5.97 \times 10^{-5}$ \\
\hline $2.90 \times 10^{-5}$ & $4.53 \times 10^{-5}$ & $1.26 \times 10^{-5}$ & $5.48 \times 10^{-5}$ & $3.62 \times 10^{-6}$ & $6.03 \times 10^{-5}$ \\
\hline $3.35 \times 10^{-5}$ & $5.22 \times 10^{-5}$ & $1.11 \times 10^{-5}$ & $4.83 \times 10^{-5}$ & $3.35 \times 10^{-6}$ & $5.58 \times 10^{-5}$ \\
\hline $3.34 \times 10^{-5}$ & $5.21 \times 10^{-5}$ & $1.36 \times 10^{-5}$ & $5.91 \times 10^{-5}$ & $2.62 \times 10^{-6}$ & $4.37 \times 10^{-5}$ \\
\hline $3.04 \times 10^{-5}$ & $4.75 \times 10^{-5}$ & $1.32 \times 10^{-5}$ & $5.74 \times 10^{-5}$ & $3.04 \times 10^{-6}$ & $5.07 \times 10^{-5}$ \\
\hline $3.34 \times 10^{-5}$ & $5.21 \times 10^{-5}$ & $1.22 \times 10^{-5}$ & $5.30 \times 10^{-5}$ & $3.34 \times 10^{-6}$ & $5.57 \times 10^{-5}$ \\
\hline $3.34 \times 10^{-5}$ & $5.21 \times 10^{-5}$ & $1.12 \times 10^{-5}$ & $4.87 \times 10^{-5}$ & $3.50 \times 10^{-6}$ & $5.83 \times 10^{-5}$ \\
\hline $3.50 \times 10^{-5}$ & $5.47 \times 10^{-5}$ & $1.11 \times 10^{-5}$ & $4.83 \times 10^{-5}$ & $2.91 \times 10^{-6}$ & $4.85 \times 10^{-5}$ \\
\hline $2.91 \times 10^{-5}$ & $4.55 \times 10^{-5}$ & $1.18 \times 10^{-5}$ & $5.13 \times 10^{-5}$ & $2.79 \times 10^{-6}$ & $4.83 \times 10^{-5}$ \\
\hline $3.55 \times 10^{-5}$ & $5.55 \times 10^{-5}$ & $1.53 \times 10^{-5}$ & $6.65 \times 10^{-5}$ & $2.85 \times 10^{-6}$ & $4.75 \times 10^{-5}$ \\
\hline $3.88 \times 10^{-5}$ & $6.06 \times 10^{-5}$ & $1.13 \times 10^{-5}$ & $4.91 \times 10^{-5}$ & $3.01 \times 10^{-6}$ & $5.02 \times 10^{-5}$ \\
\hline $3.73 \times 10^{-5}$ & $5.83 \times 10^{-5}$ & $1.34 \times 10^{-5}$ & $5.83 \times 10^{-5}$ & $2.93 \times 10^{-6}$ & $4.88 \times 10^{-5}$ \\
\hline $3.76 \times 10^{-5}$ & $5.88 \times 10^{-5}$ & $1.49 \times 10^{-5}$ & $6.48 \times 10^{-5}$ & $2.69 \times 10^{-6}$ & $4.48 \times 10^{-5}$ \\
\hline $3.57 \times 10^{-5}$ & $5.58 \times 10^{-5}$ & $1.15 \times 10^{-5}$ & $5.00 \times 10^{-5}$ & $3.21 \times 10^{-6}$ & $5.35 \times 10^{-5}$ \\
\hline $3.22 \times 10^{-5}$ & $5.03 \times 10^{-5}$ & $1.21 \times 10^{-5}$ & $5.26 \times 10^{-5}$ & $3.33 \times 10^{-6}$ & $5.55 \times 10^{-5}$ \\
\hline $3.28 \times 10^{-5}$ & $5.13 \times 10^{-5}$ & $1.08 \times 10^{-5}$ & $4.70 \times 10^{-5}$ & $3.32 \times 10^{-6}$ & $5.53 \times 10^{-5}$ \\
\hline $3.22 \times 10^{-5}$ & $5.03 \times 10^{-5}$ & $1.32 \times 10^{-5}$ & $5.74 \times 10^{-5}$ & $3.36 \times 10^{-6}$ & $5.60 \times 10^{-5}$ \\
\hline
\end{tabular}

